\title{
Focal chemotherapy of brain tumours using semipermeable membranes
}

\author{
H. A. WILKINSON, P. KORNBLITH, AND S. WEEMS \\ From Harvard Neurosurgery, Beth Israel Hospital and Massachusetts General Hospital, \\ Boston, Massachusetts, USA
}

SUMMARY Semipermeable silastic rubber membranes can be used to diffuse focally a variety of chemicals including antitumour drugs. Of the eight drugs tested, in vitro tests showed the best diffusion with dactinomycin, mithramycin, and oncovin, and the poorest diffusion with bleomycin, fluorouracil, and thiotepa. Biological testing was performed with mithramycin, dactinomycin, and oncovin using tissue cultures of human glioblastoma and subcutaneous implants of mouse ependymoblastoma. All three drugs caused rapid tissue culture cell death with direct injection, and impeded tumour growth when given intraperitoneally. Dactinomycin by silastic diffusion proved more effective than mithramycin against tissue cultures, but neither drug had a significant effect against local tumours treated with implanted drug capsules. Silastic diffusion of oncovin reduced tumour sizes significantly ipsilateral to the implant, compared to contralateral implants and to untreated controls.

At a time when tumour chemotherapy generally is gaining in scope and effectiveness, chemotherapy of brain tumours remains disappointing. Most chemotherapeutic agents do not enter brain tissue readily, and are toxic if injected intrathecally. Methotrexate is an exception since it can safely be injected intrathecally, but it is of only limited potency against brain tumours (Rubin et al., 1966; Norrell and Wilson, 1967; Wilson and Norrell, 1969). Currently the nitrosourea drugs $\mathrm{BCNU}$ and $\mathrm{CCNU}$ are receiving clinical trials against brain tumours. These drugs do enter the central nervous system readily but seem to have a beneficial effect in only about $40 \%$ of brain tumours (Wilson and Hoshino, 1969; Fewer et al., 1972; Posner and Shapiro, 1975).

This study was undertaken to explore the possibility of focal diffusion of chemotherapeutic agents directly into brain tumours or the tumour bed over a prolonged period of time. If the method can be shown to be safe and effective, it could be used as postoperative adjunctive therapy, with the device being implanted at the time of primary resection of a

Read before the 27th Annual Conference on Engineering in Medicine \& Biology, Philadelphia, Penn., 8 October 1974, and before the New England Neurosurgical Society Meeting, Evergreen Valley, Maine, 7 March 1975.

Supported by NIH Grant No. 5-R01-CA-13897, National Cancer Institute.

Accepted 9 November 1976 tumour for long-term diffusion of chemotherapeutic agents.

\section{Methods}

In vitro studies of drug diffusion through silastic rubber membranes were done first, using loops of silastic tubing of measured length immersed in testtubes containing water. Quantities of drug diffused were determined for each water bath sample by quantitative spectrophotometry.

The second phase of study consisted of two types of biological testing. In the first of these, drug diffusion was tested against tissue cultures of human glioblastoma. In the second, diffusion was tested against subcutaneously implanted mouse ependymoblastoma. Initial studies have focused on mithramycin, dactinomycin, and oncovin.

A total of 100 tissue culture flasks of human glioblastoma from an established cell line (supplied by Dr Paul Kornblith of the Massachusetts General Hospital) were studied. Before sterilisation, a 7.5 centimetre length of silastic tubing was placed across the bottom of each flask with its ends externalised through sealed openings in the flask walls. Nutrient mixture F10 (HAM) was changed aseptically every other day, and cultures were examined daily for visual semiquantitation of cell death. Although morphological changes usually preceded cell death, 
actual cell lysis or separation of all cells from the culture flask was taken as the end point of $100 \%$ kill. After cultures were well-established, they were challenged by each drug to be tested, either as a single injection directly into the medium, or by filling the silastic tubing to permit slow diffusion. Control flasks with silastic tubing, but no drug, were maintained with each group of flasks tested.

The ependymoblastoma for subcutaneous implantation in CH-3 mice was obtained from a strain maintained by Dr Nicholas Papadakis at the Boston City Hospital. Tumour removed aseptically from a 'reservoir' mouse was mixed four parts tumour: seven parts D5/Ringer's lactate: one part bicillin in a glass tissue homogeniser; $0.25 \mathrm{ml}$ of homogenate was then injected into each flank of all mice. Control mice either had no silastic implants or a unilateral implant containing no drug. Tests of systemic drug toxicity were done with four intraperitoneal injections of one drug at 48 hour intervals. All mice on silastic chemotherapy received bilateral tumour implants, and a unilateral implant of a $2.5 \mathrm{~cm}$ length of silastic tubing containing $0.04 \mathrm{ml}$ of the drug and tied closed at both ends. Drug effectiveness was assessed in terms of tumour size after a two week survival.

\section{Results}

In vitro studies showed the best rates of diffusion for dactinomycin, mithramycin, and oncovin, while least satisfactory diffusion was observed with bleomycin, fluorouracil, and thiotepa.

\section{MITHRAMYCIN STUDIES}

Twenty tissue culture flasks served as controls of various types, both with and without silastic tubing containing no active drug. In these controls, colonies could be maintained for three to four weeks. Mithramycin directly injected into the medium caused cell death in two to four days at concentrations as low as $0.01 \mu \mathrm{g} \cdot \mathrm{ml}^{-1}$ final dilution (Fig. 1). Mithramycin diffusion into the flasks through silastic rubber tubules proved disappointing when tissue culture medium changes were continued every other day, even with increased drug concentrations. Such medium changes resulted in an interrupted accumulation of mithramycin in the medium. It was possible to demonstrate a more striking antitumour effect by obviating the washout of mithramycin from the culture medium. This was accomplished by parallel diffusion of mithramycin into a reservoir of stock culture medium at the same time as diffusion was being carried out in the tissue culture flask. With each change of medium, new medium from the diffused flask was introduced so that continuously increasing levels of mithramycin were achieved. With this

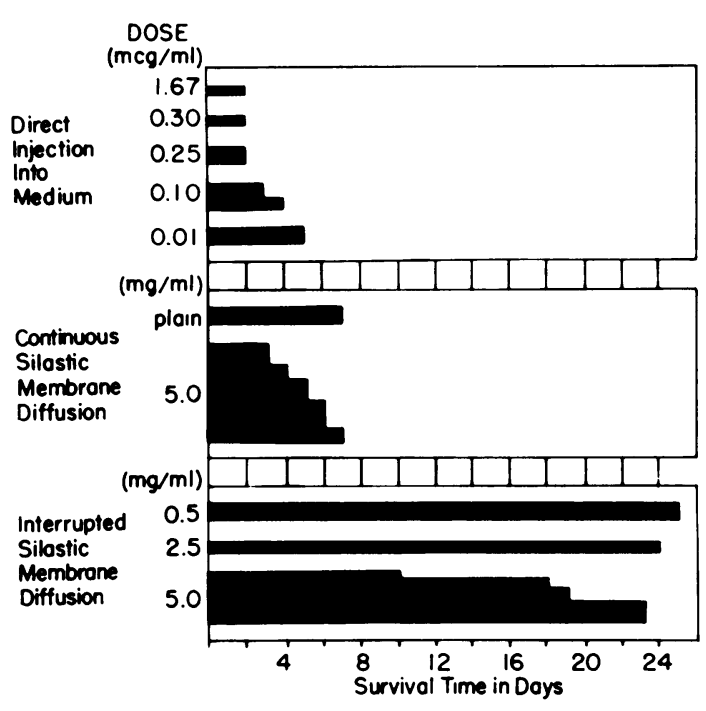

Fig. 1 Studies of human glioblastoma tissue cultures treated with mithramycin directly and by silastic membrane diffusion. Bar width denotes number of culture flasks in each group. 'Plain' indicates cultures exposed to parallel diffused medium only, with silastic membrane in flask containing no drug (see text).

technique, cell death could be consistently obtained in five to seven days.

Mithramycin was then studied against subcutane ously implanted ependymoblastoma in $121 \mathrm{CH}$ mice with 42 controls. Animals were observed over an arbitrary 14 day period after implantation, since the average survival of untreated mice with bilateral subcutaneous tumour implants varied from 10 to 20 days. Mice with subcutaneous silastic implants without tumour tolerated the devices well with no mortality (Table 1). Control mice with bilateral tumour implants, with and without unilateral silastic implants, showed similar survival rates, with tumour development reaching approximately equal size in both groups. With unilateral silastic and bilateral tumour implantation, each tumour mouse served as its own control. No significant difference in tumour size was observed from right to left when the silastic implants contained no active drug $(\mathrm{P}>0.90)$.

Mithramycin administered intraperitoneally in a group of 20 control mice at doses of 10 and $50 \mu \mathrm{g}$ was well tolerated. In the presence of bilateral tumours, the larger intraperitoneal dose of $50 \mu \mathrm{g}$ was poorly tolerated. Only two of 11 animals survived 13 days, but they exhibited marked inhibition of tumour 5 growth. Seven of 11 mice which did survive with $10 \mu \mathrm{g} \cong$ doses did show suggestive, but not statistically significant, reduction in tumour size. Ten control mice with 
Table 1 Mithramycin therapy of subcutaneous mouse ependymoblastoma

\begin{tabular}{|c|c|c|c|c|}
\hline \multirow{2}{*}{ Test group } & \multirow{2}{*}{$\begin{array}{l}\text { Number of } \\
\text { Animals }\end{array}$} & \multirow{2}{*}{$\begin{array}{l}\% \text { survival } \\
(2 w k)\end{array}$} & \multicolumn{2}{|c|}{ Tumour size in ml (Mean $+S D)$} \\
\hline & & & $\operatorname{Right}(R x) \dagger$ & Left $(n \circ R x)$ \\
\hline \multicolumn{5}{|l|}{ Controls } \\
\hline No tumour. No Rx. Silastic & 5 & 100 & - & - \\
\hline Bitumour. No Rx. No silastic & 22 & 69 & $1.97 \pm 0.68$ & $2.19 \pm 0.77$ \\
\hline Bitumour. No Rx. Silastic & 15 & 87 & $1.92+0.90$ & $1.99 \pm 0.69$ \\
\hline \multicolumn{5}{|l|}{ Intraperitoneal $\mathbf{R x}$} \\
\hline \multicolumn{5}{|l|}{ No tumour. No silastic: } \\
\hline $10 \mu \mathrm{g} \times 4$ & 10 & 100 & - & - \\
\hline $50 \mu \mathrm{g} \times 4$ & 10 & 90 & - & - \\
\hline \multicolumn{5}{|l|}{ Bitumour. No silastic: } \\
\hline $1.0 \mu \mathrm{g} \times 4$ & 4 & 75 & $2.86 \pm 1.00$ & $2.46 \pm 0.98$ \\
\hline $2.5 \mu \mathrm{g} \times 4$ & 10 & 70 & $1.94 \pm 0.93$ & $2.07 \pm 0.66$ \\
\hline $10 \mu \mathrm{g} \times 4$ & 11 & 72 & $1.54 \pm 0.76$ & $1.48 \pm 0.60$ \\
\hline $50 \mu \mathrm{g} \times 4$ & 11 & 0 & $0.30 \pm 0.10^{*}$ & $0.25 \pm 0.05^{*}$ \\
\hline \multicolumn{5}{|l|}{ Silastic diffusion $\mathbf{R x}$} \\
\hline \multicolumn{5}{|l|}{ No tumour. Silastic: } \\
\hline $5 \mathrm{mg} / \mathrm{ml}$ & 10 & 100 & - & - \\
\hline \multicolumn{5}{|l|}{ Bitumour. Silastic: } \\
\hline $0.5 \mathrm{mg} / \mathrm{ml}$ & 20 & 95 & $1.75 \pm 0.85$ & $1.34 \pm 0.85$ \\
\hline $5.0 \mathrm{mg} / \mathrm{ml}$ & 25 & 48 & $1.67 \pm 0.44$ & $2.12 \pm 0.73$ \\
\hline
\end{tabular}

*Based on two mice which survived 13 days.

$\dagger \mathbf{R x}=$ antitumour chemotherapy.

unilateral silastic implants containing mithramycin tolerated these devices well.

A total of 45 mice was studied with bilateral tumour implants and unilateral mithramycin silastic implants. These animals generally tolerated their implantations well with lower drug concentrations, and showed smaller tumour size on the side of the silastic implant with greater drug concentrations, although reduction in tumour growth did not reach statistical significance $(\mathrm{P}=0.5)$.

\section{DACTINOMYCIN STUDIES}

Human glioblastoma tissue cultures showed that direct injection produced rapid tumour kills with final drug concentrations as low as $0.002 \mu \mathrm{g} . \mathrm{ml}^{-1}$ (Fig. 2). With dactinomycin concentrations of 0.5 mg. $\mathrm{ml}^{-1}$ inside the silastic diffusion tubule, disappointingly little effect was obtained by diffusion. With the concentration of dactinomycin increased in the diffusion tubing to $1.0 \mathrm{mg} \cdot \mathrm{ml}^{-1}$, tissue kill levels were improved, with survivals limited to eight to 11 days, even with alternate day changes of fresh culture medium.

In vivo studies of dactinomycin against subcutaneous mouse ependymomas were completed in a series of 121 mice. Calculations on the basis of in vitro data suggest that approximately $6.5 \mu \mathrm{g}$ of dactinomycin should diffuse through a $25 \mathrm{~mm}$ length of silastic tubing over a 14 day planned mouse survival, assuming that in vivo diffusion rates do not differ too greatly from in vitro rates. Intraperitoneal therapy in mice with no tumour with 1.0 and $10.0 \mu \mathrm{g}$ doses each administered four times was rather well tolerated.
With intraperitoneal therapy in animals with bilateral subcutaneous tumour implants, mice failed to survive with doses of 50 and $10 \mu \mathrm{g}$, each administered four times. Mice receiving $5 \mu \mathrm{g}$ intraperitoneally suffered an $80 \%$ mortality, though the two survivors had no detectable residual tumour. A series of 25 mice receiving $1.0 \mu \mathrm{g}$ showed good survival and significantly smaller tumour size $(P<0.01)$. Since our calculated silastic delivery falls within this range of total drug exposure, these results were considered encouraging.

Five mice with subcutaneous silastic implants containing dactinomycin but no tumour tolerated these implants well with no premature mortalities. A series of 25 mice were studied with bilateral tumour implants and unilateral implants of silastic containing dactinomycin. Survival in this group was depressed over expected survival with bilateral tumour implants alone. Although tumour sizes were generally smaller ipsilateral to the silastic implants, overall no clear impairment of tumour growth was demonstrated.

\section{ONCOVIN STUDIES}

Direct injection into tissue culture flasks of human glioblastoma caused death in 48 hours with a 1.0 $\mu$ g. $\mathrm{ml}^{-1}$ dose (Fig. 3). Silastic diffusion of oncovin at a concentration of $5.0 \mathrm{mg} \cdot \mathrm{ml}^{-1}$ caused cell death in all flasks in eight to 13 days. Cells in control flasks could be maintained for 21 to 25 days.

Intraperitoneal injections of four doses of $100 \mu \mathrm{g}$ oncovin into 10 mice with no tumours proved fatal to all (Table 3). Four intraperitoneal injections of 


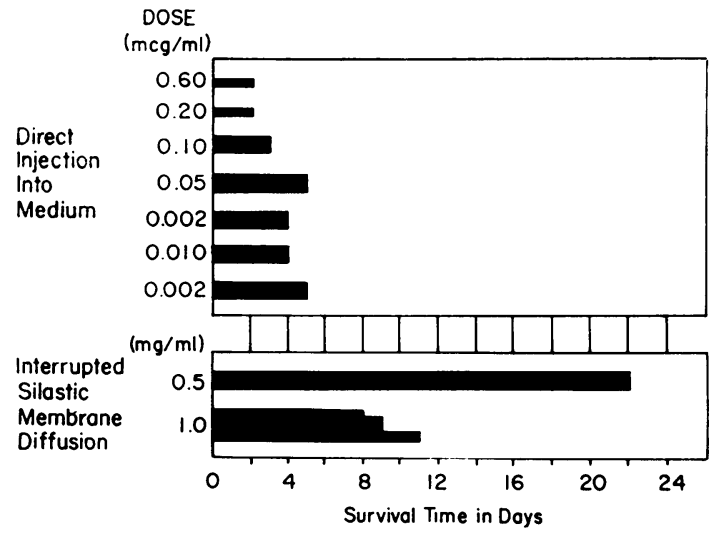

Fig. 2 Studies of human glioblastoma tissue cultures treated with dactinomycin directly and by silastic membrane diffusion. Bar width denotes number of culture flasks in each group.

oncovin killed all mice with $50 \mu \mathrm{g}$ doses, and killed four of 20 mice with 25 and $15 \mu \mathrm{g}$ doses, though none of these animals had detectable amounts of residual tumour. Thirty-one of 34 mice survived with four doses of 1 to $10 \mu \mathrm{g}$, and all demonstrated significant reduction in tumour size $(\mathrm{P}<0.001)$.

Fifty-five mice with bilateral subcutaneous ependymomas were treated with unilateral silastic implants containing either 100 or $400 \mu \mathrm{g}$ of oncovin, and 36 of these mice survived. While reduction in tumour size did not reach statistical significance for tumours contralateral to the silastic implant (average reduc-

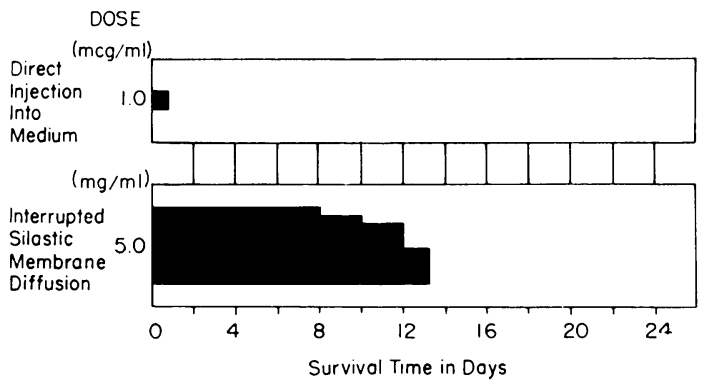

Fig. 3 Studies of human glioblastoma tissue cultures treated with oncovin directly and by silastic membrane diffusion. Bar width denotes number of culture flasks in each group.

tion $=6 \%, \mathrm{P}=0.7$ ), reduction in tumour size was highly significant for tumours ipsilateral to the silastic implant (average reduction $=28 \%$ ), as compared to both controls $(P=0.002)$, and to contralateral tumours $(\mathrm{P}=0.02)$.

\section{Discussion}

Semipermeable membranes of silastic rubber have been used in various situations in humans and animals for the slow and prolonged diffusion of a variety of biologically active compounds (Long and Folkman, 1967). Capsules containing hormones and subcutaneously implanted in animals have been used to prevent ovulation or to prolong lactation over many months (Tatum, 1970). Urinary bladder catheters

Table 2 Dactinomycin therapy of subcutaneous mouse ependymoblastoma

\begin{tabular}{|c|c|c|c|c|}
\hline \multirow{2}{*}{ Test group } & \multirow{2}{*}{$\begin{array}{l}\text { Number of } \\
\text { animals }\end{array}$} & \multirow{2}{*}{$\begin{array}{l}\% \text { survival } \\
(2 w k)\end{array}$} & \multicolumn{2}{|c|}{ Tumour size in ml $($ Mean $+S D)$} \\
\hline & & & $R i g h t(R x) \dagger$ & Left $($ no $R x)$ \\
\hline \multicolumn{5}{|l|}{ Controls* } \\
\hline No tumour. No Rx. Silastic & 5 & 100 & - & - \\
\hline Bitumour. No Rx. No silastic & 22 & 69 & $1.97 \pm 0.68$ & $2.19 \pm 0.77$ \\
\hline Bitumour. No Rx. Silastic & 15 & 87 & $1.92+0.90$ & $1.99 \pm 0.69$ \\
\hline \multicolumn{5}{|l|}{ Intraperitoneal $\mathbf{R x}$} \\
\hline \multicolumn{5}{|l|}{ No tumour. No silastic: } \\
\hline $1.0 \mu \mathrm{g} \times 4$ & 3 & 100 & - & - \\
\hline $10 \mu \mathrm{g} \times 4$ & 7 & 72 & - & - \\
\hline \multicolumn{5}{|l|}{ Bitumour. No silastic: } \\
\hline $0.05 \mu \mathrm{g} \times 4$ & 24 & 67 & $1.27 \pm 0.59$ & $1.21 \pm 0.47$ \\
\hline $0.1 \mu \mathrm{g} \times 4$ & 14 & 86 & $1.90 \pm 0.76$ & $1.79 \pm 0.65$ \\
\hline $1.0 \mu \mathrm{g} \times 4$ & 25 & 60 & $1.08+0.81$ & $0.74+0.79$ \\
\hline $5.0 \mu \mathrm{g} \times 4$ & 10 & 20 & 0 & 0 \\
\hline $10 \mu \mathrm{g} \times 4$ & 4 & 0 & 0 & $\mathbf{0}$ \\
\hline $50 \mu \mathrm{g} \times 4$ & 4 & 0 & 0 & 0 \\
\hline \multicolumn{5}{|l|}{ Silastic diffusion $\mathbf{R} \times$} \\
\hline \multicolumn{5}{|l|}{ No tumour. Silastic: } \\
\hline $1.0 \mathrm{mg} / \mathrm{ml}$ & 5 & 100 & - & - \\
\hline \multicolumn{5}{|l|}{ Bitumour. Silastic: } \\
\hline $1.0 \mathrm{mg} / \mathrm{ml}$ & 25 & 56 & $1.90 \pm 0.53$ & $2.59 \pm 0.81$ \\
\hline
\end{tabular}

${ }^{*}$ Same control mice as in Table 1 .

$+\mathbf{R} \mathbf{x}=$ antitumour chemotherapy. 
Table 3 Oncovin therapy of subcutaneous mouse ependymoblastoma

\begin{tabular}{|c|c|c|c|c|}
\hline \multirow{2}{*}{ Test group } & \multirow{2}{*}{$\begin{array}{l}\text { Number of } \\
\text { animals }\end{array}$} & \multirow{2}{*}{$\begin{array}{l}\% \text { survival } \\
(2 w k)\end{array}$} & \multicolumn{2}{|c|}{ Tumour size in $m l($ Mean $+S D)$} \\
\hline & & & $\operatorname{Right}(R x)^{*}$ & Left (no $R x)$ \\
\hline \multicolumn{5}{|l|}{ Matched controls } \\
\hline Bitumour. No Rx. No silastic & 11 & 91 & $1.98 \pm 0.48$ & $1.49 \pm 0.72$ \\
\hline \multicolumn{5}{|l|}{ Intraperitoneal $\mathbf{R x}$} \\
\hline \multicolumn{5}{|l|}{ No tumour. No silastic: } \\
\hline $100 \mu \mathrm{g} \times 4$ & 10 & 0 & 0 & 0 \\
\hline \multicolumn{5}{|l|}{ Bitumour. No silastic: } \\
\hline $50 \mu \mathrm{g} \times 4$ & 4 & $\mathbf{0}$ & 0 & 0 \\
\hline $25 \mu \mathrm{g} \times 4$ & 10 & 60 & 0 & 0 \\
\hline $15 \mu \mathrm{g} \times 4$ & 10 & 100 & 0 & 0 \\
\hline $10 \mu \mathrm{g} \times 4$ & 4 & 50 & $0.05 \pm 0.00$ & $0.05 \pm 0.00$ \\
\hline $5 \mu \mathrm{g} \times 4$ & 20 & 95 & $0.37 \pm 0.48$ & $0.60 \pm 0.80$ \\
\hline $1 \mu \mathrm{g} \times 4$ & 10 & 100 & $0.40 \pm 0.34$ & $0.35 \pm 0.39$ \\
\hline \multicolumn{5}{|l|}{ Silastic diffusion $\mathbf{R x}$} \\
\hline \multicolumn{5}{|l|}{ Bitumour. Silastic: } \\
\hline $100 \mu \mathrm{g}$ total & 40 & 65 & $1.32 \pm 0.73$ & $1.55 \pm 0.57$ \\
\hline $400 \mu \mathrm{g}$ total & 15 & 67 & $1.16 \pm 0.72$ & $2.17 \pm 0.91$ \\
\hline Combined groups & 55 & 65 & $1.29 \pm 0.70$ & $1.77 \pm 0.74$ \\
\hline
\end{tabular}

* $\mathrm{Rx}=$ antitumour chemotherapy.

have been designed for human use, with antibiotics impregnated into the rubber for slow chronic release over many weeks (Wepsic, 1971). Experimentally, silastic rubber membranes have been used to induce general anaesthesia through an intravenously implanted loop (Folkman et al., 1966, 1968, 1969; Folkman and Mark, 1968). Silastic rubber semipermeable membranes were studied initially in our laboratory, in conjunction with Drs Vernon Mark and Judah Folkman, in the form of 'chemodes' chronically implanted in the brains of experimental animals (Wilkinson et al., 1971, 1973). These devices have been shown to be impenetrable to bacteria and non-toxic to brain tissue. Studies previously performed in our laboratory, and studies in other laboratories, have documented slow diffusion through these membranes of a variety of compounds, including some antitumour drugs (Sehgal et al., 1972; Schmidt et al., 1972; Rosenblum et al., 1973).

Because of the blood brain barrier, most chemotherapeutic agents enter brain tissue poorly except at blood levels toxic to other organs and tissues. Semipermeable membranes, chronically implanted into brain tumours or into their vicinity, offer the possibility of bypassing this barrier. Such devices have received a preliminary clinical trial in the form of chemodes chronically implanted in the brains of human patients for diffusion of biogenic amines (Mark et al., 1969). Animal studies in our laboratory have shown safe diffusion of various agents through intracerebrally implanted silastic capsules, and others have shown similar diffusion of chemotherapeutic agents to be locally effective against subcutaneously implanted mouse tumours (Rosenblum, et al., 1973).
Many agents, discarded from clinical use because of systemic toxicity, have nevertheless been shown to have significant antitumour effectiveness against brain tumours in tissue culture and in extracerebral implants. Most chemotherapeutic agents have low lipid solubility, and pass through the blood brain barrier and through silicone rubber membranes slowly. Agents with high lipid solubility, such as the nitrosoureas, cross the blood brain barrier readily into brain tumours. Their lipid solubility should also allow rapid diffusion through silastic, with high local concentrations. Unfortunately, we have shown with other drugs (Wilson et al., to be published), and have tentatively confirmed with some chemotherapy drugs, that the more lipid soluble a chemical is, the more destructive it is to brain tissue locally. Thus slow diffusion of a relatively lipid insoluble drug may offer the benefit of greater tissue safety while permitting the application to brain tumours of effective chemotherapy agents ordinarily excluded by the blood brain barrier.

By using silastic membranes it may be possible to diffuse chemotherapeutic agents focally into brain tumours, avoiding the extracerebral toxic effects of these drugs. Cerebral gliomas are diffusely invasive tumours so that total resection of tumour can almost never be accomplished without sacrifice of unacceptably large amounts of still functioning though tumour infiltrated brain. By implanting silastic membrane devices for chronic antitumour therapy around the margins of gross tumour resections it is hoped that further growth of known residual tumour can be slowed or halted. 


\section{References}

Fewer, D., Wilson, C. B., Boldrey, E. B., Enot, K. J., and Powell, M. R. (1972). The chemotherapy of brain tumors: Clinical experience with carmustine (BCNU) and vincristine. Journal of the American Medical Association, 222, 549-551.

Folkman, J., Long, D. M., and Rosenbaum, R. (1966). Silicone rubber: A new diffusion property useful for general anesthesia. Science, 154, 148-149.

Folkman, J. and Mark, V. H. (1968). Diffusion of anesthetics and other drugs through silicone rubber: Therapeutic implications. Transactions of the New York Academy of Sciences, 30, 1187-1195.

Folkman, J., Mogul, T., and Winsey, S. (1968). Anesthesia by diffusion through silicone rubber. Anesthesiology, 29, 410-418.

Folkman, J., Reiling, W., and Williams, G. (1969). Chronic analgesia by silicone rubber diffusion. Surgery, 66, 194-200.

Long, D. M. and Folkman, J. (1967). Polysiloxane carrier for controlled release of drugs and other agents. US Patent 3,249.996 18 October 1966). Cancer, 66, $5759 \mathrm{~g}$.

Mark, V., Folkman, J., Ervin, F., and Sweet, W. (1969). Focal brain suppression by means of a silicone rubber chemode. Journal of Neurosurgery, 30, 195-199.

Norell, H. and Wilson, C. (1967). Brain tumor chemotherapy with methotrexate given intrathecally. Journal of the American Medical Association, 201, 93-95.

Posner, J. B. and Shapiro, W. R. (1975). Brain tumorscurrent status of treatment and its complications. Archives of Neurology (Chicago), 31, 589-603.

Rosenblum, M. L., Bowie, D. L., and Walker, M. D. (1973). Diffusion in vitro and in vivo of 1-(2-chloroethyl)-3-(trans-4-methylcyclohexyl)-1-nitrosourea from silicone rubber capsules, a potentially new mode of chemotherapy administration. Cancer Research, 33, 906-914.
Rubin, R. C., Ommaya, A. K., Henderson, E. S., Bering, E. A., and Rall, D. P. (1966). Cerebrospinal fluid perfusion for central nervous system neoplasms. Neurology (Minneapolis), 16, 680-692.

Schmidt, V., Zapol, W., Prensky, W., Wonders, T., Wodinsky, I., and Kitz, R. (16-18 April 1972). Continuous cancer chemotherapy: Nitrosourea diffusion through implanted silicone rubber capsules. Read before American Society of Artificial Internal Organs Meeting, Seattle, Wash.

Sehgal, L. R., deRois, M. E., Feinberg, H., and Szanto, P. (1972). Interstitial cancer chemotherapy with silastic implants. Federation Proceedings, 31, 575.

Tatum, H. (1970). Fertility control and acceptability in women of contraceptive steroids released in micro quantities from subcutaneous silastic capsules. Contraception, 1, 253.

Wepsic, J. G. (10 August 1971). Catheter having antibacterial substance therein provided with means permitting slow release of said substance. US Patent $3,598,127$.

Wilkinson, H. A., Mark, V. H., and Wilson, R. B. (1971). Sleep induced by focal brain suppression using anesthetic gases. Experimental Neurology, 30, 30-33.

Wilkinson, H. A., Mark, V. H., Wilson, R. B., and Patel, P. P. (1973). The toxicity of general anesthetics diffused directly into the brain. Anesthesiology, 38, 478-481.

Wilson, C. B. and Hoshino, T. (1969). Current trends in the chemotherapy of brain tumors with special reference to glioblastoma. Journal of Neurosurgery, 31, 589-603.

Wilson, C. B. and Norrell, H. A. (1969). Brain tumor chemotherapy with intrathecal methotrexate. Cancer, 23, 1038-1045.

Wilson, R. B., Wilkinson, H. A., Esmaili, M., and Patel, P. (1976). Comparison of volatile anesthetic agents for reversible focal brain suppression in cats. Submitted for publication, 1976. 